\section{Green issues fuel funding}

\section{Palmer Station}

IF the case of the US Antarctic programme is any guide, the principal effect of greenhouse warming and ultraviolet radiation may be wild, unpredictable growth. Riding the coattails of international concern over ozone and global change, the US Antarctic effort has unexpectedly found itself at the focus of the decade's hottest environmental research, a windfall that has resulted in a tripling of funding over the past ten years.

Since 1980, the National Science Foundation (NSF) programme has seen its Antarctic budget increase from $\$ 55$ million to $\$ 175$ million, a spurt in growth that will have an immediate impact in a host of new facilities and improvements at the main US bases.

At McMurdo Station, 750 miles from the South Pole on the rocky shores of the Ross Sea, a 46,000-square-foot, three-storey science laboratory is nearly finished. It will join newly renovated dormitories and administrative buildings to finish a five-year renaissance for the 1,200-person base, until recently best known for its mud and hard life.

The South Pole's Amundsen-Scott Station is also seeing a flurry of construction. Twenty years of drifting snow have half-buried the 50-foot aluminium geodesic dome that houses most of the station, causing uneven pressures and structural damage. This year, engineers bulldozed away tons of snow, repaired parts of the dome and raised some parts by nearly a foot to level the structure and balance its loads. They believe that the repairs have extended the dome's life span by some 20 years. Work will begin later this year on a new summer base at the South Pole, to house the increasing numbers of scientists who now come to the already overcrowded station during the peak research months. And later in the decade, NSF hopes to begin construction on a new and much larger station at the Pole (see page 289).

At Palmer Station, on the relatively temperate Antarctic Peninsula, NSF is getting ready to start its first Antarctic Long-Term Ecological Research (LTER) site. Under a six-year grant, researchers at Palmer will carry out studies throughout the food web from ocean dynamics and algae growth to penguins - to try to understand how the 'carbon cycle' biology of the Antarctic oceans may affect global warming, and vice versa.

One Antarctic 'facility' that is not yet in the polar region is the Nathaniel B. Palmer, a major new research vessel with ice-breaking capacity now under construction in Louisiana. When the 300 foot, \$83.8-million vessel is first launched around the end of the year, it will join the 219-foot Polar Duke in full-time Antarctic service to complete NSF's two-ship research fleet.

NSF has no plans for further major construction once these facilities are finished. Although the Antarctic programme is ex- pected to continue growing, agency officials intend to increase research efforts not by building more structures, but by bringing in more scientists and fewer support personnel. Already an NSF 'streamlining plan' has doubled the ratio of researchers to support staff in ten years by hiring private contractors to take over any support function's from the Navy, which is notorious for its bureaucratic inefficiency and redundancy.

"The flexibility of using contractors is that we don't have to carry them when we don't need them", says one NSF official. "Using

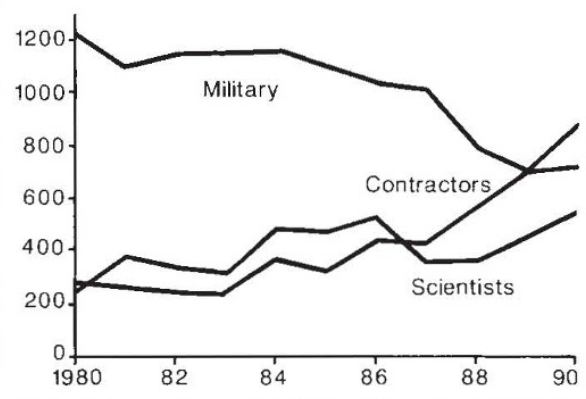

Scientists and contractors are an increasing proportion of the US contingent.

fewer people saves money, as well as wear and tear on the continent."

Another way to get more science without importing more manpower is to use automation, a trend that has come far since its beginnings in weather-monitoring stations. Automated clean-air facilities, telescopes, seismic stations, ozone-monitoring equipment and underwater sampling stations are all in use, overseen by the technicians who maintain other station equipment, or by no one at all. Scarce Antarctic slots can now be filled by other researchers whose work requires personal attention, leaving the most mindless research responsibilities to the machines.

C.A.

\section{Pole position}

\section{Washington}

US researchers will build three new telescopes at the South Pole, as part of a new centre announced last month by the NSF. The facility will be one of the 14 new research centres funded by NSF as the second phase of its three-year-old network of interdisciplinary, university-based Science and Technology Centers (STCs). Headed by astronomers from the University of Chicago, the South Pole centre will operate as a consortium, with eight university and industry partners. NSF funding will be $\$ 2$ million for 1991 , and up to $\$ 13.6$ million over the next five years.

Each of the three South Pole telescopes will focus on a fundamental problem in astronomy. The Cosmic Background Radiation Anisotropy (COBRA) Project will look for tiny (one part in a million) variations in the cosmic radiation background as part of the investigation of the origins of galaxies. A second telescope, known as the South Pole Infrared Explorer project (SPIREX), will take advantage of the South Pole's low infrared background to study the formation of stars in young galaxies.

The third project will focus on the interstellar medium in nearby galaxies. Known as the Antarctic Submillimeter Telescope/ Remote Observatory (AST/RO), the project will also measure ozone in the Earth's atmosphere.

Project members plan to operate the telescopes during the harshest winter months at the Pole, when the station is isolated from the outside world and temperatures can drop to $-100^{\circ} \mathrm{F}$.

Developing telescopes to operate in those conditions, and to follow celestial objects from a constantly moving surface, will require new technology that will help in the design of even more advanced telescopes elsewhere, centre officials say.

C.A.

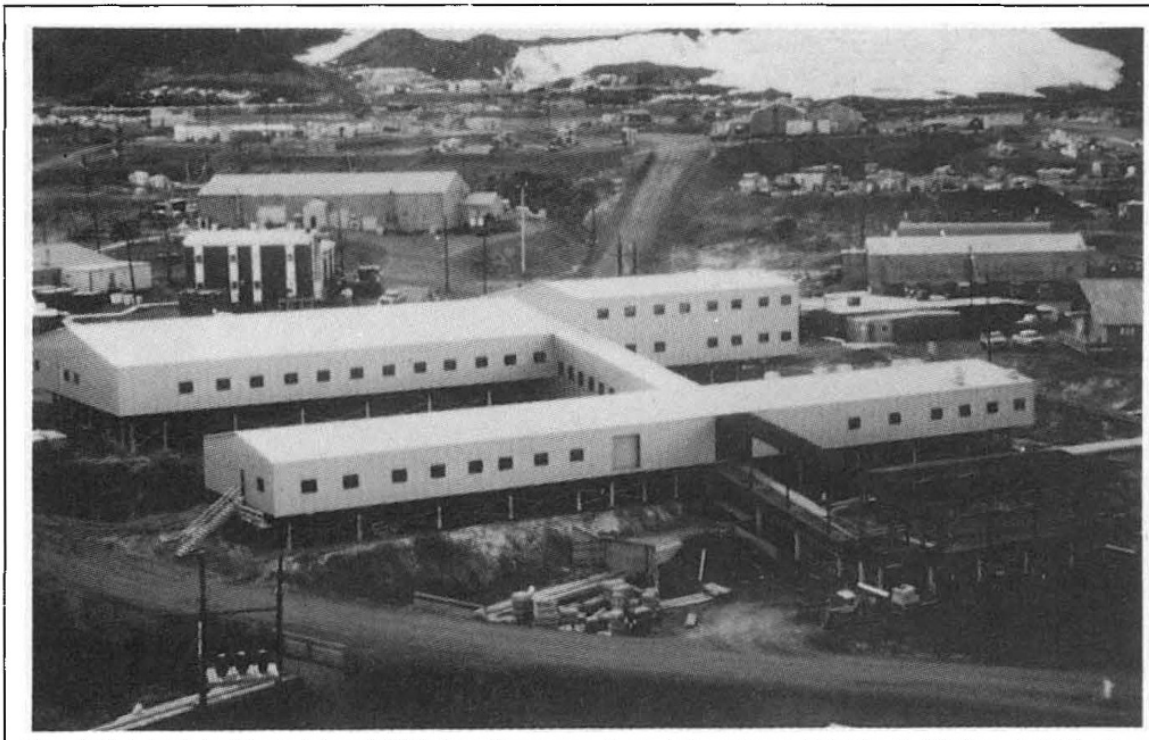

Nearly finished, in this October 1990 view, the new science laboratory at McMurdo Station. Cold Regions Research Laboratory photo by Wayne Tobiasson. 